\title{
Job Motivation and Organizational Commitment as Contributing to Employees' Burnout
}

\author{
Keren Michael \\ Department of Human Services, The Max Stern Yezreel Valley Academic College, Israel
}

\begin{abstract}
Background: Occupational burnout is a problematic phenomenon that influences both employees' and organizations' productivity. It often seems like the result of entering a workplace with high job motivation and high organizational commitment but experiencing disappointment or not getting constructive outcomes.

Aims: The present study examined how employees' perceptions regarding job motivation and organizational commitment contribute to burnout.

Method: The sample in this quantitative cross-sectional study included 400 service organizations' employees (59.7\% women; 62.4\% worked in a full-time job) aged 19-71 (Mean age=29.94; $\mathrm{SD}=10.25$ ). All participants completed self-reported questionnaires measuring job motivation, sense of organizational commitment (affective, continuance), and levels of burnout (emotional exhaustion, depersonalization, reduced personal accomplishment). Data were analyzed using IBM-SPSS (version 25) through multiple regressions.

Results: The path analysis demonstrated that all three dimensions of burnout were predicted by low job motivation and low affective commitment. In contrast, emotional exhaustion and depersonalization were predicted by high continuance commitment. Additionally, an affective commitment was predicted by high job motivation, while continuance commitment was predicted by low job motivation.

Conclusions and Implications: According to the results, the employees' perceptions within the organization contribute to their feelings of burnout. Specifically, there are differences in the states of commitment and their effects. Therefore, efforts to promote employees' affective commitment should be encouraged to help relieve work burnout, negatively influencing both employees and the organization.
\end{abstract}

Keywords: motivation, commitment, burnout, organizations. 